\title{
The Seemingly Preferred Cosmic Frame
}

\section{Fulvio Melia}

Department of Physics, The Applied Math Program, and Department of Astronomy, The University of Arizona, Tucson AZ 85721, USA

E-mail: fmelia@email.arizona.edu

Submitted: 8 December 2021

\begin{abstract}
The Universe appears to have a 'preferred' frame of reference, within which the cosmic microwave background is completely isotropic. Earth's motion through this relic radiation field is measurable, and some have viewed this type of observation as a possible contradiction of Einstein's principle of relativity. Several experiments have been proposed (and some have been carried out) to test for violations of Lorentz invariance when transforming out of this background frame, e.g., based on expected velocity differences in a Michelson-Morley type of probe. But what exactly is this cosmic frame? Is it truly an absolute frame attached to the Universe, or is it an observer-dependent construction? Such possibilities represent very different Physics, of course. In this paper, we work through these issues carefully, without the introduction of unsubstantiated assumptions. We show that the preferred frames originate through general (not special) relativity, and are indeed observer-dependent. Their existence does not at all conflict with Einstein's fundamental hypothesis of special relativity.
\end{abstract}

Keywords: General Relativity, Observational Cosmology, Mathematical and Relativistic Aspects of Cosmology

\section{Introduction}

It is widely believed that the Universe has a 'preferred' frame of reference, selected by the Cosmic Microwave Background (CMB) or, more specifically, the geometry of the last scattering surface (LSS), where the CMB photons emerge out of the optically-thick medium [1]. Some observational support for this view is provided by the fact that any peculiar motion of our Galaxy relative to the CMB produces a measurable Doppler effect responsible for the prominent dipole moment seen in the temperature anisotropies [2].

One of the earliest arguments in favor of a cosmic preferred frame was made by Greber \& Blatter [3], who considered a front-back asymmetry in the intensity and pressure sensed by an observer moving 'through' the CMB. The interaction of matter with the CMB radiation, they concluded, would tend to bring the matter to rest in the preferred frame where the radiation is isotropic.

This perspective gives rise to some discomfort, however, because the existence of such a preferred frame of reference seems to contradict the fundamental hypothesis of special relativity, which Einstein referred to as the principle of relativity [4]. For example, a violation of this principle could affect the universality of the speed of light, especially its constancy and isotropy, and may even impact the viability of special relativity itself.

Yet violations of Lorentz invariance in a broader context, and their implied existence of preferred reference frames (not nec- essarily just that of the CMB) could explain differences in the behavior of photon propagation from various high-energy $\gamma$-ray sources (MAGIC Collaboration [5]; Fermi Collaboration [6]), and various proposals have been advanced for finding these violations $[7,8]$ to address this possibility. Effects associated with transformations in and out of a preferred CMB frame may also alter the particle dispersion relation, $E^{2}=F(p, m)$, which presumably reduces to the standard form, $F(p, m) \rightarrow p^{2} c^{2}+m^{2} c^{4}$ in the latter, but deviates from it in others [7]. It is not always evident, however, that the discussion of a preferred frame, and possible tests of Lorentz-invariance violations associated with it, are consistently clear on what exactly is meant by such a frame. For example, in their proposal to observe an 'etherdrift,' in which one tries to measure a small difference in the velocity of light in different directions, Consoli \& Pluchino [9] consider the possibility that the CMB frame might actually be an absolute Universal frame, with some physically sustainable vacuum structure.

But whether conceived within a legitimate physical framework or not, all experiments to detect a violation of Lorentz invariance associated with a preferred CMB frame appear to have produced null results thus far. In one of the more interesting and tangible attempts, De Angelis et al. [10] studied over 62 million well-reconstructed $K_{S}^{0} \rightarrow \pi^{+} \pi^{-}$decays recorded by the KLOE detector (KLOE Collaboration [11]) at the DAФNE accelerator in Frascati, in order to measure differences in the $K_{S}^{0}$ meson lifetimes parallel $\left(\tau_{+}\right)$and antiparallel $\left(\tau_{-}\right)$to Earth's motion with respect to the CMB reference frame. No differences were 
found, with a $95 \%$ confidence (upper) limit of $0.98 \times 10^{-3}$ on any possible asymmetry $\left|\tau_{+}-\tau_{-}\right| /\left(\tau_{+}+\tau_{-}\right)$.

The existence of a preferred frame for relativity is thus an open question. Clearly, Earth's motion 'through' the CMB is detectable. But what exactly is this cosmic frame? Is it truly an absolute frame for the Universe, or is it perhaps attached to us? And if the latter, does that mean that different observers would thus see different 'absolute' frames?

The outcome of this type of discussion inevitably impacts whether Lorentz invariance is violated, which in turn affects several modern physical theories. It is possible that the Lorentz symmetry is broken even if the cosmic frame is observer dependent, given that the CMB has a special, unique distribution in this frame, though it is unquestionably more obvious that such effects could be manifested if the cosmic frame is unique and attached to the Universe rather than the observer.

The motivation for even asking this question is that the reason such a frame exists is typically not addressed by this discussion. We tend to take it for granted that some preferred frame must be present because the CMB somehow indicates this must be true. But in spite of the fact that its existence is broadly acknowledged, there appear to be conflicting views concerning whether it defines a special Lorentz frame as background, through which all of the various observers are moving, or whether it may be defined as the 'free-falling' frame at every given spacetime point within the cosmos. The latter possibility is not as easy to understand, and therefore receives less attention by the Physics community.

A clear appreciation of this universal frame, how it originates, and how it impacts our physical theories, is critical to its proper implementation within relativity theory [12]. One might be tempted, e.g., to abolish such foundational tenets as the 'principle of relativity' and the constancy of the speed of light [13]. Or one might instead prefer to modify those principles and assume from the outset that such a preferred inertial frame is the only one in which the two-way speed of light (i.e., the average lightspeed from source to observer and back) is isotropic, while it must by default then be anisotropic in any other relatively moving frame. The latter approach has been taken, e.g., by ref. [14], in which the existence of a preferred frame is incorporated into the framework of the special relativity at the expense of losing the freedom to assign the one-way speed of light in Einstein's theory. This can then lead to the development of an anisotropic relativistic kinematics, with an embedded anisotropic propagation of light [15]. And further still, this may impact how we view local inertial frames and the equivalence principle in general relativity. Without venturing too far down this path, such developments can clearly affect whether the interval remains invariant (probably not), and whether one must then determine how to conformally modify it under a transformation of coordinates between different frames [16].

It is interesting to note, in this regard, that the present interpretation of an accelerating Universe as due to the influence of dark energy $[2,17]$ may be misdirected. Instead, a relativity theory with a preferred frame may 'correct' the redshift-distance relation in such a way that it modifies the deceleration parameter, possibly explaining why we infer an acceleration when in fact there is none [14].

In this paper, our principal focus will be to identify the ori- gin of this preferred frame, which can then help us demonstrate whether it is truly attached to the Universe, or whether it is merely dependent on the observer. These two possibilities represent very different Physics. We shall work through these issues carefully and pedagogically, avoiding any unnecessary (i.e., unsubstantiated) assumptions. We shall find that what we know today in cosmology already offers us the insights we need to answer these questions in a straightforward manner.

\section{Background}

\subsection{Specific Intensity}

Consider a group of particles occupying a tiny spread in position and momentum at some given time. In a frame comoving with the particles, these occupy a spatial volume element

$$
d^{3} \mathbf{x}^{\prime} \equiv d x^{\prime 1} d x^{\prime 2} d x^{\prime 3}
$$

and a momentum volume element

$$
d^{3} \mathbf{p}^{\prime} \equiv d p^{\prime 1} d p^{\prime 2} d p^{\prime 3} .
$$

In the infinitesimal limit, these particles have no spread in energy because the contribution to ${p^{\prime}}^{0}$ from $\mathbf{p}^{\prime}$ in the rest frame is quadratic and thus vanishes to first order.

This group of particles occupies an element of phase space

$$
d \mathcal{V}^{\prime} \equiv d^{3} \mathbf{p}^{\prime} d^{3} \mathbf{x}^{\prime} .
$$

Given the above conditions, including the fact that there is no spread in energy, an observer outside of the rest frame will see a spatial volume element that transforms inversely to the corresponding volume in momentum space, and so

$$
d \mathcal{V} \equiv d^{3} \mathbf{p} d^{3} \mathbf{x}=d \mathcal{V}^{\prime},
$$

i.e., the volume in phase space is invariant. Although this result is well known, its formal proof is actually non-trivial, given that one must carefully compare the change in momentum-space volume under a Lorentz transformation to the corresponding change in spatial volume. A detailed discussion of this procedure may be found in ref. [18].

This simple argument is based on the behavior of particles with mass, but note that there is actually no reference to mass in Equation (4). One may therefore consider this equation in the limit when mass $\rightarrow 0$, and see that it applies to photons as well. It thus follows that the phase-space density of radiation must also be invariant, because the number of photons is a countable quantity and is a prime example of a Lorentz scalar:

$$
f \equiv \frac{d \mathcal{N}}{d \mathcal{V}}=\text { invariant }
$$

Now, if $u_{v}(\Omega)$ represents the energy density of the radiation per unit frequency $v$ and solid angle $d \Omega$, we have

$$
u_{v}(\Omega) d v d \Omega=(h v f) p^{2} d p d \Omega,
$$

where we have written $d^{3} \mathbf{p}=p^{2} d p d \Omega$ in the given frame. By definition, the specific radiative intensity is

$$
I_{\nu}(\Omega) \equiv u_{\nu}(\Omega) c,
$$


and therefore

$$
\frac{I_{v}}{c} d v d \Omega=(h v f) p^{2} d p d \Omega .
$$

Further, since $p=h v / c$, we evidently must have

$$
\frac{I_{v}}{v^{3}}=\frac{h^{4}}{c^{2}} f
$$

which brings us to our final result,

$$
\frac{I_{v}}{v^{3}}=\text { invariant }
$$

This is a textbook result, and quite familiar to high-energy astrophysicists. But we have reconsidered it here because of its key importance to everything else that follows in this paper.

\subsection{Blackbody Spectrum}

Next, suppose we have a blackbody spectrum in some frame $K$, written as

$$
I_{v}=B_{v}(T) \equiv \frac{2 h v^{3} / c^{2}}{\exp (h v / k T)-1},
$$

where $v$ and $T$ are the frequency and temperature in $K$. According to Equation (10), an observer in some other frame $K_{0}$, will then see an intensity $I_{v_{0}}$, such that

$$
\frac{I_{v_{0}}}{v_{0}{ }^{3}}=\frac{I_{v}}{v^{3}}=\frac{2 h / c^{2}}{\exp (h v / k T)-1} .
$$

The functional form on the righthand side is that of a blackbody again, though still in terms of the frequency $v$ and temperature $T$ in $K$. In the $K_{0}$ frame, however, the intensity invariant is $I_{v_{0}} / v_{0}^{3}$, written in terms of the new coordinates. For consistency, one should then also use the new frequency in the denominator of Equation (12), so that $\exp (h v / k T) \rightarrow \exp \left(h v_{0} / k\left[v_{0} / v\right] T\right)$. And the most 'natural' interpretation of this expression is therefore that the observer in $K_{0}$ again sees a blackbody shape, though with a transformed temperature

$$
T_{0} \equiv\left(\frac{v_{0}}{v}\right) T
$$

which allows one to recast Equation (12) into the form

$$
I_{v_{0}}=B_{v_{0}} \equiv \frac{2 h v_{0}^{3} / c^{2}}{\exp \left(h v_{0} / k T_{0}\right)-1} .
$$

In other words, a blackbody spectrum in one frame looks like a blackbody spectrum in every other frame, except that its inferred temperature is observer dependent. This too is critical to the central theme of this paper.

\subsection{The Cosmological Context}

Thus, if the surface of last scattering (LSS) and recombination are at redshift $z_{\text {LSS }}$, here on Earth we observe a cosmic microwave background intensity given by Equation (14) with a temperature

$$
T_{0}=\frac{T\left(z_{\mathrm{LSS}}\right)}{\left(1+z_{\mathrm{LSS}}\right)}
$$

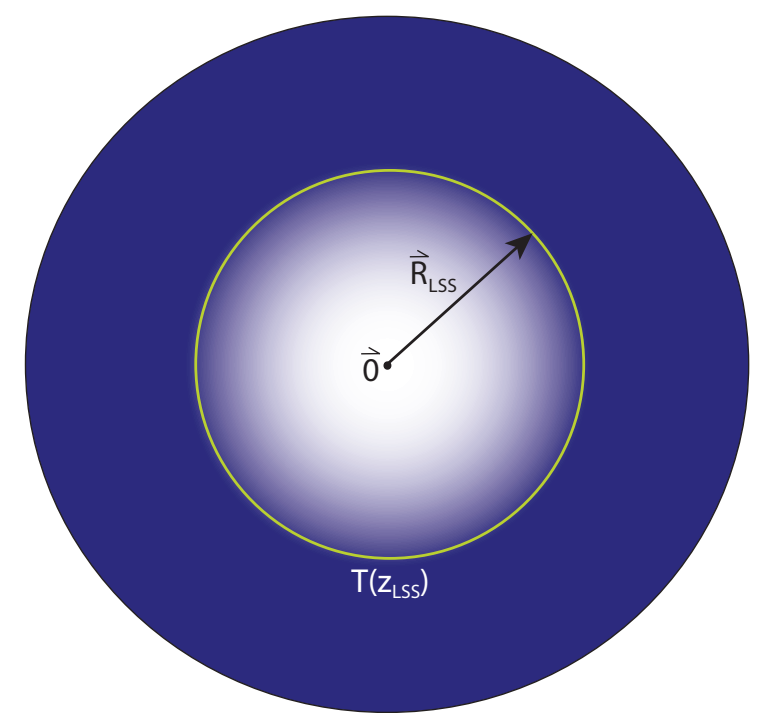

Figure 1. The Cosmological Principle postulates that the Universe is homogeneous and isotropic about us (located at the origin). The proper distance $R_{\mathrm{LSS}}$ to the last scattering surface (LSS), and the blackbody temperature $T\left(z_{\mathrm{LSS}}\right)$ at that redshift, should thus be independent of direction. The CMB originates from a spherical shell at $R_{\mathrm{LSS}}$ (we ignore the actually thickness of the emission region, which is much smaller than $R_{\mathrm{LSS}}$ ), and propagates towards us through an optically thin medium. For simplicity, we assume the Universe is completely opaque beyond the LSS.

(see fig. 1), where we have used the definition of redshift,

$$
1+z \equiv \frac{v}{v_{0}}
$$

in terms of the frequency $(v)$ of the blackbody radiation at the emitter (in this case, the LSS) and its corresponding value $\left(v_{0}\right)$ locally.

\subsection{The Cosmological Principle}

Modern cosmology is founded on the Cosmological Principle (CP), which postulates that the Universe is homogeneous and isotropic about every point, at least on scales [19] larger than $\sim 300 \mathrm{Mpc}$. This principle is the basis for the highly symmetric Friedmann-Lemaître-Robertson-Walker (FLRW) metric used to describe the cosmic spacetime [20], written as

$$
d s^{2}=c^{2} d t^{2}-a^{2}(t)\left[\frac{d r^{2}}{\left(1-k r^{2}\right)}+r^{2}\left(d \theta^{2}+\sin ^{2} \theta d \phi^{2}\right)\right] .
$$

In this expression, $t$ is the proper time measured by a comoving observer, and the corresponding radial $(r)$ and angular $(\theta$ and $\phi)$ coordinates in this frame remain "fixed" for all particles in the cosmos. The spatial curvature constant $k$ is +1 for a closed universe, 0 for a flat, open universe, and -1 for an open universe. The universal expansion is accounted for by the expansion factor $a(t)$, which is solely a function of time, not position.

The CP suggests that our proper distance $R_{\mathrm{LSS}} \equiv a(t) r_{\mathrm{LSS}}$ to recombination is the same in every direction and that the CMB temperature $T\left(z_{\text {LSS }}\right)$ should be independent of our chosen line-of-sight (see fig. 1). Of course, we have now confirmed [2] that $T_{0}$ exhibits tiny fluctuations - at the level of one part 
in $10^{5}$ - but these are presumably associated with primordial quantum effects unrelated to any possible violation of the CP. In other words, the LSS must be a sphere centered on us, with a blackbody temperature $T\left(z_{\text {LSS }}\right)$ given solely by the LSS redshift $z_{\text {LSS }}$ and nothing else.

\subsection{Cosmological Redshift}

In order to fully comprehend the role played by the LSS shown in figure 1 in establishing our preferred frame of reference, we need to discuss the origin of redshift $z$ in Equation (16), when this relation is applied to the cosmological setting. The issue of whether or not cosmological redshift is a new, third type of redshift, distinct from the better known kinematic and gravitational effects typically found in other applications of general relativity, has been debated for many decades. On the one hand, its interpretation as an effect due to the "stretching" of space has been based on the expression

$$
1+z=\frac{a\left(t_{0}\right)}{a\left(t_{e}\right)}
$$

where $t_{0}$ and $t_{e}$ are, respectively, the cosmic time at which the radiation is observed and that at which it was emitted [21]. This formulation doesn't look like any of the other forms of redshift we have encountered before, and seems to suggest that cosmological redshift grows as the Universe expands, i.e., as $a(t)$ increases with time.

The counterargument has been that Equation (18) appears to be valid in only one coordinate system, so the 'stretching of space' interpretation is probably not real. Various authors have attempted to demonstrate this more formally (see, e.g., refs. [22, 23]) but, given the complexity of the problem, had been forced to rely on various forms of approximation. A more complete demonstration that cosmological redshift is in fact just the traditional lapse function in general relativity was presented about a decade ago [24]. In this formulation, $z$ is just the product of the well-understood Doppler and gravitational shifts in frequency. In one particular coordinate system, $z$ depends just on the expansion factor (Eq. 18), but this is merely a manifestation of the high degree of symmetry in FLRW, and should not be viewed as evidence supporting the notion that space itself is expanding.

As we shall see shortly, the Doppler shift is easy to calculate for any cosmological model. The gravitational redshift is more difficult because it relies on our ability to track the change in energy of a photon as it propagates to us along its null geodesic. This procedure is most easily carried out when the FLRW metric has a time-independent spacetime curvature, because the photon's energy then remains constant along its trajectory. Regardless of how the gravitational redshift is calculated, however, the kinematic contribution to the lapse function (Eqn. 20 below) is identical in every case. For illustration, we consider the method of finding the cosmological redshift at the LSS using the lapse function for those FLRW spacetimes that may be written in terms of coordinates $(c T, R, \theta, \phi)$ for which the metric coefficients are static.

There are exactly six, and only six, such cases [25, 26], including the Milne Universe and de Sitter space. This approach includes three essential steps: (i) first, one must find $(c T, R, \theta, \phi)$ via an appropriate transformation of the coordinates $(c t, r, \theta, \phi)$ in the comoving frame, where the FLRW metric takes the form given in Equation (17); (ii) The FLRW metric written in terms of the transformed coordinates $(c T, R, \theta, \phi)$ is used to calculate the time dilation, $d t / d T$, at the LSS relative to the proper time in a local free-falling frame; (iii) Finally, one calculates the apparent time dilation, different from the time dilation at the emitter's location due to the motion of the source (i.e., due to the recession velocity of the LSS shell), which alters the relative arrival times of the photon's wave crests. The two latter steps are well known in general relativity [21]; the most complicated step is often the first, though by now we know how to complete the transformation for all six static FLRW metrics [24].

Using the lapse function, the ratio of the frequency of the light observed (at the origin) to that emitted at the LSS (at time $t_{e}$ ), may be written

$$
\frac{v_{0}}{v_{e}}=\left.\left(1+\frac{v_{R}}{c}\right)^{-1} \frac{d t}{d T}\right|_{t_{e}},
$$

where the subscript $t_{e}$ on the right-hand side emphasizes the fact that this expression must be evaluated at the emitter. The kinematic correction introduced by the motion of the source relative to the observer, which accounts for the difference between the apparent time dilation and its counterpart at the source - and is independent of how the gravitational redshift is calculated-is the factor

$$
D \equiv \frac{1}{1+v_{R} / c},
$$

written in terms of the proper velocity (proper distance per unit proper time) seen in this frame along the line-of-sight:

$$
v_{R} \equiv \sqrt{\frac{g_{R R}}{g_{T T}}} \frac{d R}{d T},
$$

where $g_{\alpha \beta}$ are the FLRW metric coefficients written in terms of the coordinates $(c T, R, \theta, \phi)$.

\section{The Preferred Cosmic Frame}

With the pieces we have assembled in $\S$ II, we may now proceed to understand why the CMB defines a unique frame of reference at the origin. If the Universe were a vacuum, any pair of frames moving at constant velocity relative to each other would be inertial and completely Lorentz invariant under a transformation of physical quantities from one to the other. But the Universe is not a vacuum, and we do not live in a flat cosmic spacetime which means that, at our position, there is a unique free-falling frame-one that satisfies the $\mathrm{CP}$ and within which there is net zero gravitational acceleration. It is general (not special) relativity that leads us to this conclusion, formally via the so-called Local Flatness Theorem. So before we discuss the preferred frame itself, let us first consider how this theorem, together with the $\mathrm{CP}$, selects a unique inertial frame at each observer's location.

\subsection{The Local Flatness Theorem}

In general relativity, the Local Flatness Theorem $[21,27]$ is a formal representation of the equivalence principle. It says that 
in the neighborhood of any spacetime point $X^{\mu}$ in the (usually curved) frame of reference $x^{\mu}$ there must exist a local inertial frame, against which one may measure the acceleration in $x^{\mu}$. This local inertial frame, which we shall label $\xi^{\mu}$, is in free fall at $X^{\mu}$.

Since the CP holds that the Universe looks identical no matter where we place the observer, let us for convenience therefore place ourselves at the origin of our coordinates $\left(x^{\mu}\right)$ and (without loss of generality) let us orient the $\xi^{\mu}$ frame so that its origin coincides with ours. Of course, the point $X^{\mu}$ lies at this common origin. The key to understanding the implications of this theorem on the frame $\xi^{\mu}$ is the requirement that these coordinates must satisfy the equations [21]

$$
\frac{\partial^{2} \xi^{\alpha}}{\partial x^{\mu} \partial x^{\nu}}=\Gamma_{\mu \nu}^{\lambda} \frac{\partial \xi^{\alpha}}{\partial x^{\lambda}} .
$$

These relations express the transformation from our frame, with a known spacetime curvature represented by the Christoffel symbols $\Gamma_{\mu \nu}^{\lambda}$, to one (i.e., $\xi^{\mu}$ ) in which the spacetime curvature is zero. They are exact, with no approximation or assumption, other than the validity of the equivalence principle.

We will not reproduce all the steps required to solve for the four components $\xi^{\mu}$ here. The details have been published elsewhere [28] and are easy to follow. The $\alpha=0$ component in Equation (22), with $\mu=v=0$, simply gives

$$
\xi^{0}=c t,
$$

while the $\alpha=1$ component shows that

$$
\xi^{i}=B\left(x^{i}\right) c t,
$$

where both the spatial coordinates $x^{i}$ and $\xi^{i}$ are written in the Cartesian system, and $B\left(x^{i}\right)$ is a function solely of $x^{i}$. Then, the same $\alpha=0$ component in Equation (22) with $\mu=v=1$ shows that

$$
B\left(x^{i}\right)=B_{0} e^{x^{i} / c t_{0}},
$$

where $B_{0}$ is a constant and $t_{0}$ is the current age of the Universe.

We thus see that the inertial frame identified by Equation (22) has Cartesian velocity components

$$
\frac{d \xi^{i}}{d \xi^{0}}=\left(\frac{1}{c t_{0}} \frac{d x^{i}}{d(c t)}+\frac{1}{c t}\right) \xi^{i} .
$$

And this velocity therefore goes to zero at $\xi^{i}=X^{i}=0$ where the observer is located. Notice that both the Local Flatness Theorem and the inclusion of the CP were necessary to arrive at this conclusion, i.e., that the FLRW metric defines a unique freefalling frame at the observer's location. There are, of course, numerous other inertial frames at this location too, but they all have a non-zero velocity relative to this one, and the Universe does not look isotropic from the perspective of another observer within them.

\subsection{The Observer's Preferred Frame}

Now, in the context of FLRW, every point in the Universe recedes from this local frame at a proper velocity proportional to its proper distance away from the origin,

$$
\frac{d R}{d t} \hat{r}=H \vec{R}
$$

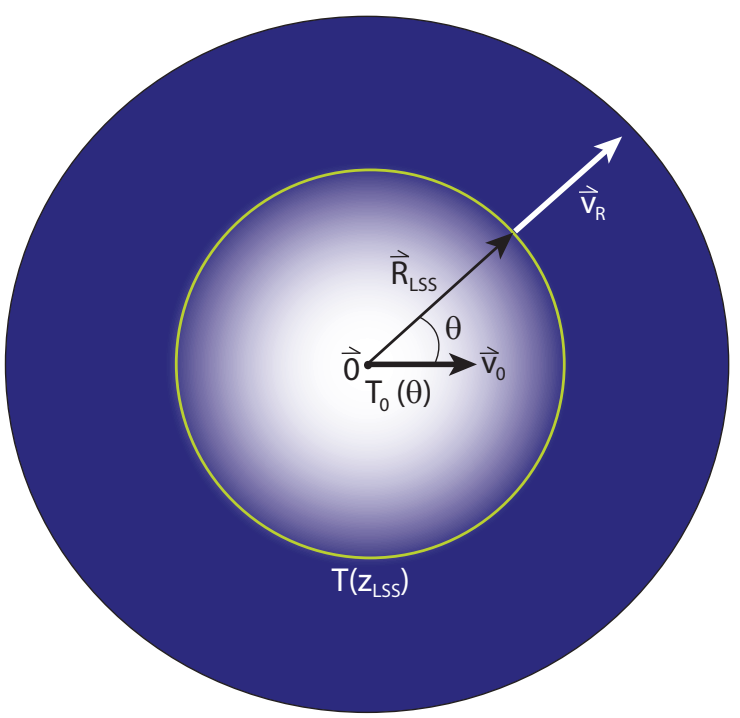

Figure 2. Same as figure 1, except now the geometry of the LSS is being viewed from the perspective of a frame moving at constant velocity $\mathbf{v}_{0}$ relative to the origin. While the proper distance $R_{\mathrm{LSS}}$ to the last scattering surface (LSS), and the blackbody temperature $T\left(z_{\text {LSS }}\right)$ at that redshift, are still independent of direction, the transformed temperature $T_{0}$ measured by the observer (Eq. 15) becomes a function of the angle, $\theta$, between $\mathbf{v}_{0}$ and the proper velocity $\mathbf{v}_{R}$ along the line-ofsight at the LSS.

where the Hubble parameter is given in terms of the expansion factor $a(t)$ :

$$
H \equiv \frac{1}{a} \frac{d a}{d t} .
$$

Such an intuitive picture of regularity in the FLRW spacetime stems from Weyl's postulate [29], which says that the worldlines of all galaxies form a 3-bundle of non-intersecting geodesics orthogonal to a foliated sequence of spacelike hypersurfaces. An important consequence of this picture is that any two points in the FLRW spacetime must therefore be receding from each other at a speed proportional to their separation [20].

Thus, the local free-falling frame at our location, positioned at $\overrightarrow{0}$, is distinguished from all other frames by the fact that the average velocity (not speed) of recession of all the spacetime points around us is exactly zero. This is the sole cosmic 'landmark' one can use to identify a position and velocity. And according to the $\mathrm{CP}$, the $\mathrm{CMB}$ must therefore look identical to us in all directions from our perspective in this frame. The flexibility of choosing among different inertial frames in special relativity therefore does not even enter this discussion. On the other hand, a frame moving at $\mathbf{v}_{0} \neq 0$ at the origin does not satisfy this requirement, as we shall now see.

Assuming that $v_{0} \ll v_{R}$, we may approximate the proper velocity of an emitting element on the LSS relative to the observer moving with respect to $\overrightarrow{0}$ (see fig. 2) to be

$$
v_{\mathrm{LSS}} \approx v_{R}-v_{0} \cos \theta \text {, }
$$

instead of $v_{\text {LSS }}=v_{R}$ in the case of zero velocity. (Note that by assuming $v_{0} / v_{R} \ll 1$, we can ignore the tiny corrections one ought to include from a change in angle under a Lorentz transformation.) Thus, we need to slightly modify Equation (19) to 
read

$$
\frac{v_{0}}{v_{e}}=\left.\left(1+\frac{v_{R}-v_{0} \cos \theta}{c}\right)^{-1} \frac{d t}{d T}\right|_{t_{e}}
$$

and, using a Binomial expansion and keeping only the leading order terms, we find that

$$
\frac{v_{0}}{v_{e}}=\left(\frac{v_{0}}{v_{e}}\right)_{v_{0}=0}\left(1+\frac{v_{0}}{v_{R}} \cos \theta\right) \text {. }
$$

Finally, using Equation (15), we get

$$
T_{0}(\theta)=\left\langle T_{0}\right\rangle\left(1+\frac{v_{0}}{v_{R}} \cos \theta\right),
$$

where

$$
\left\langle T_{0}\right\rangle \equiv \frac{T\left(z_{\mathrm{LSS}}\right)}{\left(1+z_{\mathrm{LSS}}\right)} .
$$

Equation (32) represents a pure dipole pattern of the observed CMB temperature in terms of the angle $\theta$ between the line-ofsight and the direction of our Galaxy's motion, measured in the preferred frame of the LSS (see fig. 2), which also happens to correspond to our unique free-falling frame imposed by the Local Flatness Theorem at our location. By way of interest, this kinematic dipole effect has been measured with various satellite instruments, suggesting that our solar system is moving at speed $v_{0}=369 \pm 1 \mathrm{~km} \mathrm{~s}^{-1}$ in the direction $(l, b)_{\mathrm{CMB}}=\left(264^{\circ}, 48^{\circ}\right)$ in Galactic coordinates [30]. The angular variations in $T_{0}$ have an amplitude $\sim 3 \mathrm{mK}$, compared to $T_{0}$ itself which has a measured value (Planck Collaboration 2016) of $2.72548 \pm 0.00057 \mathrm{~K}$.

This kinematic interpretation of the dipole pattern in $T_{0}(\theta)$ is the most widely held view, but we should nevertheless acknowledge a possible caveat with this argument. Our 'measurement' of the velocity $v_{0}$ is done indirectly, by comparing the angle-dependent temperature $T_{0}(\theta)$ to its average value $\left\langle T_{0}\right\rangle$. Regardless of its value, however, we always see thermal radiation, as shown in Equation (14). Though we believe it to be unlikely, the situation may therefore be that $v_{0}=0$ and the $\mathrm{CMB}$ itself has intrinsic variations that create a dipole pattern in $T_{0}(\theta)$ when we observe it. This could happen, for example, if the surface where the CMB radiation is produced is not a perfect sphere around us. Then the temperature we measure could vary with angle due to the redshift (or distance) dependence of the radiation field. This would seem highly contrived to us, so we do not consider it to be a probable explanation, but we should be aware that this possibility exists.

Assuming that our interpretation of $v_{0}$ is correct, and that it does indeed identify a special frame with $v_{0}=0$, does this violate Einstein's 'principle of relativity,' and thereby call into question the viability of special relativity itself? Of course not. First of all, the Universe is not a vacuum, so the application of special relativity to the selection of inertial frames at the observer's location is invalid. Second, the uniqueness of our preferred cosmic frame (located at $\overrightarrow{0}$ in fig. 2 ) is due to the singular nature of local free-falling frames in a curved spacetime. One may wish to consider other frames moving at constant velocity with respect to $\overrightarrow{0}$, but at the risk of sacrificing isotropy and violating the Cosmological Principle.

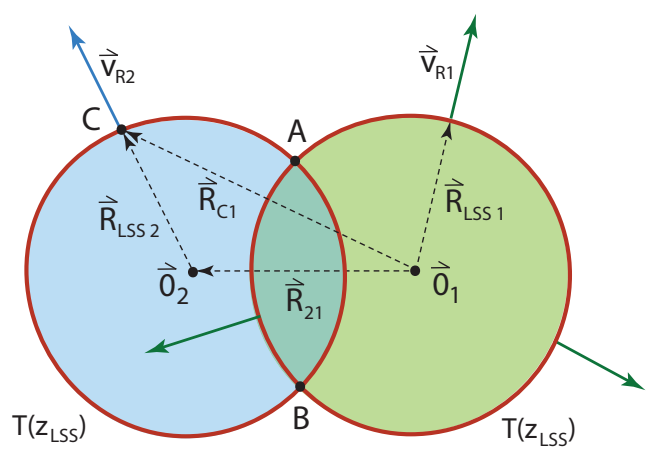

Figure 3. Two observers, one at $0_{1}$ and the other at $0_{2}$, a proper distance $R_{21}$ away, each sees a spherically-symmetric LSS surrounding their location, receding at proper speeds $v_{R 1}$ and $v_{R 2}$, respectively. The Universe is homogeneous on any given time slice $t$, so the physical conditions are identical throughout the cosmos. Therefore, $R_{\mathrm{LSS} 2}=R_{\mathrm{LSS} 1}, v_{R 2}=v_{R 1}$, and the blackbody temperature $T\left(z_{\mathrm{LSS}}\right)$ is uniform everywhere on the LSS surfaces. But whereas the Universe is opaque beyond the LSS centered on $0_{1}$ for the first observer, it is opaque only beyond the LSS centered on $\mathrm{O}_{2}$ for the second, in spite of the fact that these two particular spherical regions overlap between $\mathrm{A}$ and B. The LSS geometry seen by the second observer is identical to that seen by the first, but their preferred frames are distinctly different. In fact, $0_{2}$ is receding from $0_{1}$ at proper speed $d R_{21} / d t=H R_{21}$.

The story is not yet complete, however, because an additional level of confusion sometimes arises with the perceived validity of the relativity principle in the cosmic context, which is sometimes questioned as follows: Does all of this mean that the Universe really has just one preferred frame and, if so, against what independent background could such a solitary frame be identified? The answer is again a resounding 'no'. The preferred frame is a local frame attached, not to the Universe as a whole, but to each individual observer. The Universe itself has no absolutely defined frame of reference. On the other hand, there could in principle be an infinite number of observers distributed throughout an infinite Universe, implying the coexistence of an infinite number of such preferred frames.

Consider the schematic diagram of two nearby observers shown in figure 3 , one located at $\overrightarrow{0}_{1}$, the other at the second origin $\overrightarrow{0}_{2}$, a proper distance $R_{21}$ away. As noted earlier, in the context of FLRW, every spacetime point at proper radius $\vec{R}_{1}=a(t) \vec{r}_{1}$ with respect to observer 1 is receding from $\overrightarrow{0}_{1}$ with a velocity $H R_{1} \hat{r}_{1}$. This includes the second observer at $\overrightarrow{0}_{2}$, who must therefore be moving to the left relative to the first at speed $d R_{21} / d t=H R_{21}$.

The first observer sees a spherically-symmetric LSS with radius $R_{\mathrm{LSS} 1}$ (surrounding the optically-thin green sphere), receding from her at proper speed $v_{R 1}$. According to the CP, the second observer sees his own spherically-symmetric LSS with radius $R_{\mathrm{LSS} 2}$ (surrounding an equally optically-thin blue sphere), receding from him at proper speed $v_{R 2}$. The homogeneity of the Universe ensures that the local physical conditions are identical throughout the cosmos, inside the spherical domains surrounded by the LSS, within the LSS shells and also throughout the exterior. But optical depth, which defines the LSS geometry, is an observer-dependent measure, based on the integrated scattering and absorption of light along each individ- 


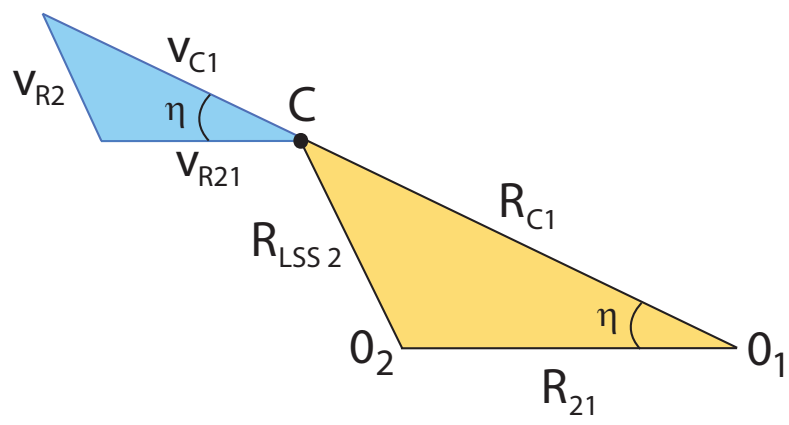

Figure 4. Vector geometry of the point C on the LSS of observer 2 in figure 3 from the perspective of $0_{1}$ and $0_{2}$. The yellow triangle relates the proper distances of these three points, while the blue does the same for their relative proper velocities.

ual's line-of-sight. Thus, the location (and shape) of the LSS for the second observer is completely independent of that of the first and, in spite of these two particular LSS shells overlapping, observer 1 sees an opaque Universe everywhere beyond her spherical LSS shell centered on $\overrightarrow{0}_{1}$, while observer 2 sees an opaque Universe only beyond his spherical LSS shell centered on $\overrightarrow{0}_{2}$. The overlap region between points $\mathrm{A}$ and $\mathrm{B}$ has no impact at all on the observers' perception of where the $\mathrm{CMB}$ originates for them individually.

In addition, one can easily confirm in the context of FLRW that the proper velocity of any given point C on the LSS of observer 2 must be receding from $\overrightarrow{0}_{2}$ at a proper velocity $\vec{v}_{R 2}=v_{R 1} \hat{r}_{2}$, just as every point on the LSS of observer 1 recedes from $\overrightarrow{0}_{1}$ with a corresponding velocity $\vec{v}_{R 1}=v_{R 1} \hat{r}_{1}$. Simply employing the cosine rule for the two triangles in figure 4 , we see that

$$
R_{\mathrm{LSS} 2}^{2}=R_{21}^{2}+R_{C 1}^{2}-2 R_{21} R_{C 1} \cos \eta,
$$

and

$$
v_{R 2}^{2}=v_{C 1}^{2}+v_{R 21}^{2}-2 v_{C 1} v_{R 21} \cos \eta,
$$

where $v_{C 1} \equiv d R_{C 1} / d t$. Thus,

$$
v_{R 2}^{2}=H^{2}\left[R_{21}^{2}+R_{C 1}^{2}-2 R_{21} R_{C 1} \cos \eta\right],
$$

which reduces to

$$
v_{R 2}=H R_{\mathrm{LSS} 2} .
$$

In other words, point $\mathrm{C}$ is receding radially from observer 2 at the same speed as the LSS of observer 1 is receding from her (i.e., $v_{R 2}=v_{R 1}$ ) because $R_{\mathrm{LSS} 2}=R_{\mathrm{LSS} 1}$. And the blackbody temperature $T\left(z_{\text {LSS }}\right)$ is uniform everywhere on the two LSS shells. The CMB and LSS geometry seen by the second observer are therefore identical to those observed by the first, though their preferred frames of reference are distinctly different. Each sits in their own locally free-falling frame, but these are receding from each other at speed $d R_{21} / d t=H R_{21}$. They are not the same frame, which should dispel any notion of a single, unique frame attached to the Universe as a whole.

\section{Conclusion}

So the Universe does have preferred frames, though these are attached to individual observers. The cosmos itself has no absolute frame of reference. In some sense, this characterization lends some support to the relativity principle, because it reaffirms our flexibility of choosing among a myriad free-falling frames-merely by deciding where in the cosmos we wish to position the origin $\overrightarrow{0}$ of our coordinates.

This distinction impacts several areas in physics, not merely our interpretation of the cosmic spacetime. For example, as we noted earlier, several experiments have been designed to search for any violation of Lorentz invariance or symmetry, both foundational elements of fundamental physics, which would question the underpinnings of well-known physical laws, such as CPT symmetry. The invariance of physical laws under the simultaneous transformations of charge conjugation (C), parity reversal $(\mathrm{P})$ and time reversal $(\mathrm{T})$ is viewed as a fundamental symmetry of nature [31], such that CPT is the only combination observed thus far to not be violated at a fundamental level. The CPT theorem states that this symmetry must hold for any Lorentz invariant local quantum field theory with a Hermitian Hamiltonian.

But these violations are predicted by certain forms of quantum gravity and string theory. They are also expected in alternative gravity theories to Einstein's general relativity. To be clear, violations of Lorentz invariance could be seen in various contexts, not just the cosmic domain. But if the Universe would indeed have had a single preferred frame, any breakdown of the Lorentz symmetry could quite directly have been visible with reference to this special frame.

Lorentz violations could take several forms, including a variation in the speed of light, either within one frame, or a transformation between inertial frames, as well as deviations from the time dilation predicted by special relativity. These effects are often estimated within the context of modified special relativity theories and and effective field theories, including the Standard-Model Extension, which introduce Lorentz and CPT violations via spontaneous symmetry breaking due to the presence of hypothetical background fields. One might see in these modified spacetimes a modification of the dispersion relation, leading to measurable differences between the speed of light and the maximum speed attainable by matter.

On the flip side, the non-existence of such a singular preferred frame makes it meaningless to talk about the violation of Lorentz invariance associated with transformations in and out of it in the context of special relativity. A test such as that proposed by Coleman \& Glashow [32], when applied to the CMB preferred frame, in which particle oscillations could be measurable due to velocity differences in a modern-day version of the Michelson-Morley experiment, may eventually reveal some effects, but their interpretation would be questionable. Experiments designed to test for such violations without taking into account the general-relativistic origin of an observer's preferred frame of reference are unlikely to be true tests of the relativity principle in flat spacetime.

As of today, several terrestrial and astronomical experiments have been carried out [7], some of which have been highlighted earlier in this paper, but no Lorentz violations have been confirmed so far, though a few positive results were reported at 
first, but not confirmed subsequently. Some recent experimental searches are described in ref. [33], and an overview of some Lorentz violating scenarios may be found in ref. [34].

Nevertheless, whether or not an anomaly is ever detected, the type of analysis we have carried out in this paper can only deepen our understanding of the cosmic spacetime [20], and we continue to marvel at the incredible beauty and elegance of the Universe we live in.

\section{Acknowledgement}

I am grateful to the Instituto de Astrofísica de Canarias in Tenerife and to Purple Mountain Observatory in Nanjing, China for their hospitality while part of this work was carried out. I am also grateful to the anonymous referees for their thoughtful and helpful suggestions to improve the presentation in this paper.

\section{References}

[1] Gawiser E Silk J 2000 Physics Reports 333245

[2] Planck Collaboration et al2016 AEA 594 A13 63pp

[3] Greber T \& Blatter H 1990 Am. J. Phys. 58942

[4] Einstein A Lorentz H S Minkowski H \& Weyl H 1952 The Principle of Relativity: A Collection of Original Memoirs on the Special and General Theory of Relativity Sommerfeld A (ed.) (Mineola, NY: Dover Publications).

[5] MAGIC Collaboration 2008 PLB 668253

[6] Fermi Collaboration 2009 Nature 462331

[7] Mattingly D 2005 Living Reviews in Relativity 8 id 5 84pp

[8] Lämmerzahl C 2006 in Ehlers J Lämmerzahl C (eds) Special Relativity Lecture Notes in Physics vol 702 (Berlin: Springer)

[9] Consoli M \& Pluchino A 2018 EPJ Plus 133 id 295 27pp

[10] De Angelis A et al2011 Il Nuovo Cimento 34323

[11] KLOE Collaboration 2011 EPJ-C 711604

[12] Will C M Nordtvedt K Jr 1972 ApJ 177757

[13] Ungar A A 1986 Phil Sci 53395

[14] Burde G I 2018 e-print arXiv:1805.10995

[15] Sonego S Pin M 2009 J Math Phys 50 042902, 28pp

[16] Kastrup H A 2008 Annalen der Physik 17631

[17] Amendola L Tsujikawa S 2010 Dark energy: theory and observations (Cambridge: Cambridge University Press)

[18] van Kampen N G 1969 Physica 43244

[19] Yadav J Bagla J S \& Nishikanta K 2010 MNRAS 4052009

[20] Melia F 2020 The Cosmic Spacetime (Oxford: Taylor \& Francis)

[21] Weinberg S 1972 Gravitation and Cosmology: Principles and Applications of the General Theory of Relativity (New York: Wiley)

[22] Bunn E F Hogg D W 2009 Am J Phys 77688

[23] Chodorowski M J 2007 MNRAS 378239

[24] Melia F 2012 MNRAS 4221418

[25] Florides P S 1980 GRG 12563

[26] Melia F 2013 CQG 30 id 155007 14pp

[27] Poisson E 2007 A Relativist's Toolkit: The Mathematics of Black-Hole Mechanics (Cambridge: Cambridge University Press)

[28] Melia F 2019 Annals of Phys. 411 id 167997 8pp
[29] Weyl H 2009 GRG 411661

[30] COBE Collaboration 1993 ApJ 4191

[31] Kostelecky V A 1998 e-print arXiv:hep-ph/9810365

[32] Coleman S \& Glashow S L 1997 PLB 405249

[33] Kostelecky V A Russell N 2011 Reviews of Modern Physics 83 11

[34] Liberati S 2013 Classical and Quantum Gravity 30133001 